\title{
Quantized conductance in atom-sized wires between two metals
}

Brandbyge, Mads; Schiøtz, Jakob; Sørensen, Mads Reinholdt; Stoltze, Per; Jacobsen, Karsten Wedel; Nørskov, Jens Kehlet; Olesen, Laurits Højgaard; Laegsgaard, E.; Stensgaard, I.; Besenbacher, Flemming

Published in:

Physical Review B

Link to article, DOI:

10.1103/PhysRevB.52.8499

Publication date:

1995

Document Version

Publisher's PDF, also known as Version of record

Link back to DTU Orbit

Citation (APA):

Brandbyge, M., Schiøtz, J., Sørensen, M. R., Stoltze, P., Jacobsen, K. W., Nørskov, J. K., Olesen, L. H., Laegsgaard, E., Stensgaard, I., \& Besenbacher, F. (1995). Quantized conductance in atom-sized wires between two metals. Physical Review B, 52(11), 8499-8514. https://doi.org/10.1103/PhysRevB.52.8499

\section{General rights}

Copyright and moral rights for the publications made accessible in the public portal are retained by the authors and/or other copyright owners and it is a condition of accessing publications that users recognise and abide by the legal requirements associated with these rights.

- Users may download and print one copy of any publication from the public portal for the purpose of private study or research.

- You may not further distribute the material or use it for any profit-making activity or commercial gain

- You may freely distribute the URL identifying the publication in the public portal 


\title{
Quantized conductance in atom-sized wires between two metals
}

\author{
M. Brandbyge, J. Schiøtz, M. R. Sørensen, P. Stoltze, K. W. Jacobsen, and J. K. Nørskov \\ Center for Atomic-scale Materials Physics and Physics Department, \\ Technical University of Denmark, DK 2800 Lyngby, Denmark \\ L. Olesen, E. Laegsgaard, I. Stensgaard, and F. Besenbacher \\ Center for Atomic-scale Materials Physics and Institute for Physics and Astronomy, \\ University of Aarhus, DK 8000 Aarhus, Denmark
}

(Received 21 April 1995)

\begin{abstract}
We present experimental and theoretical results for the conductance and mechanical properties of atom-sized wires between two metals. The experimental part is based on measurements with a scanning tunneling microscope (STM) where a point contact is created by indenting the tip into a gold surface. When the tip is retracted, a 10-20 $\AA$ long nanowire is formed. Our measurements of the conductance of nanowires show clear signs of a quantization in units of $2 e^{2} / h$. The scatter around the integer values increases considerably with the number of quanta, and typically it is not possible to observe more than up to four quanta in these experiments. A detailed discussion is given of the statistical methods used in the analysis of the experimental data. The theoretical part of the paper addresses some questions posed by the experiment: Why can conductance quantization be observed, what is the origin of the scatter in the experimental data, and what is the origin of the scaling of the scattering with the number of conductance quanta? The theoretical discussion is based on a free-electron-like model where scattering from the boundary of the nanowire is included. The configurations of the nanowires are deduced from molecular dynamics simulations, which also give information about the mechanical properties of the system. We show that such a model can account semiquantitatively for several of the observed effects. One of the main conclusions of the theoretical analysis is that, due to the plastic deformation of the nanowires formed by the STM, the typical length scale of the variations in the shape of the boundary is not an atomic radius but rather five times that value. This is the reason why scattering is sufficiently small to make conductance quantization observable by STM.
\end{abstract}

\section{INTRODUCTION}

When the length scale of an electrical conductor is reduced to less than the electron mean free path, the scattering of electrons will predominantly take place at the boundaries of the conductor. This characterizes the ballistic transport regime in which the conductance is independent of material properties and determined only by the geometry of the sample and the electron density., In recent years, there has been an increasing interest in the fundamental properties of conduction in the ballistic regime, and extensive experimental and theoretical studies have been carried out. The progress in the field has mainly been due to the development of small semiconductor devices in which a two-dimensional electron gas (2DEG) can be confined by applying a voltage to a split gate. In this way, it has been possible not only to observe ballistic transport, but also to go even a step further and explore the regime of quantized conductance. ${ }^{3-5}$ Quantization is possible if the electrons are confined by the sample boundaries or applied fields in the directions transverse to the current on a length scale of the order of the Fermi wavelength of the conducting electrons.

When semiconductors are used in ballistic transport experiments, one profits from a mean free path that can be as long as several micrometers. In the quantized regime, semiconductors have the additional advantage that the electron density is very low, implying a Fermi wavelength, which typically is two orders of magnitude larger than for metals. ${ }^{5}$ This immediately indicates that the criteria to be satisfied in order to observe conductance quantization in a metal are much more severe. It demands a constriction that can be controlled not on the submicrometer scale, as in the case of semiconductors, but rather on the subnanometer scale. However, the scanning tunneling microscope ${ }^{6}$ (STM) has already been proven to be an extremely important tool in producing and modifying structures on the subnanometer scale, ${ }^{7}$ and the possibility of using the STM in an experiment on quantized metallic conduction is, therefore, obvious.

As the tip in a STM is moved towards the surface, the attractive force between tip and sample increases rapidly, ${ }^{8}$ all the way up to the point where the attractive force gradient surpasses the spring constant of the combined system of tip, sample, and interconnecting systems. ${ }^{9}$ Then a sudden jump to contact takes place, ${ }^{10,11}$ and this will, for sufficiently clean electrodes, immediately lead to a metallic contact, which can be stretched to a connective neck by a subsequent retraction of the tip. ${ }^{12} \mathrm{~A}$ connective neck can also be produced by voltage pulses. ${ }^{44}$

The behavior of the conductance in such an atomicsized metal constriction has recently been investigated with STM in many different environments and tempera- 
ture regimes, such as at $4.2 \mathrm{~K},{ }^{13,14}$ at room temperature in ambient air, ${ }^{15-17}$ at room temperature under ultrahigh vacuum (UHV) conditions, ${ }^{18}$ and at room temperature in high vacuum with an STM complemented by a force sensor. ${ }^{19}$ In the latter study, the relation between the adhesive tip-sample force and the conductance was investigated. Another technique for creating nanoconstrictions is the mechanical break junction technique, where a metal filament is broken at low temperatures, resulting in two freshly prepared electrodes that can be brought back into contact. ${ }^{20,21}$ The very small size of the constriction in these experiments should facilitate the possibility of observing the quantization of conductance, despite the short metallic Fermi wavelength, which is comparable to atomic dimensions. Some authors have reported that when the nanoconstriction is stretched and thinned, the measured conductance is quantized in units of $2 e^{2} / h$, although with some scatter around this value, ${ }^{15-19}$ whereas in other studies, such a distribution of the conductance values is less evident, and noninteger steps have been observed..$^{20,21}$ Also from a theoretical point of view, there is still no consensus. It has been suggested that the steps in the conductance are associated with mechanical instabilities in the nanoconstrictions, ${ }^{22}$ and that they are due to a quantization of the transverse electron motion in a contact containing only a few atoms. ${ }^{15,18,23}$

In the present paper, we provide new experimental evidence for real quantized conductance at room temperature in a metal constriction drawn between an STM tip and a metal surface. Extreme care has been taken in order to make the experimental conditions as well characterized as possible. To diminish the complications with impurities, water films, etc., the experiments have been performed in UHV on well defined, clean Au single crystalline metal surfaces. Furthermore, to preserve the integrity of the surface, the constrictions have been produced by tip indentations performed as gently as possible, with a depth of 3-5 $\AA$. Since the measurements depend crucially on the detailed geometry of the individual constrictions, we do not try to make a general statement on the basis of a few measurements only, but instead we apply a statistical treatment involving a large number of measurements. This analysis demonstrates that for the present results on $\mathrm{Au}(110)$, the distribution of conductances shows clear peaks at $n 2 e^{2} / h$, where $n$ is an integer. Furthermore, the area and width of the peaks are found to decrease and increase, respectively, with increasing $n$.

The details concerning the experiment and the results are presented in the first part of the paper. The second part consists of a detailed discussion of the theory of conductance in a one-dimensional conductor. We start by giving an introduction to the simplest adiabatic theory of electron transport in a one-dimensional conductor. This treatment shows the origin of the conductance quantization. The adiabatic theory assumes that the variation of the one-electron potential is slow on the scale of the Fermi wavelength $\lambda_{F}$. Since $\lambda_{F}$ is of the order an atomic diameter, one might think that scattering from atom-sized irregularities in the point contact would destroy the conductance quantization completely. We have developed a theory, including scattering from the boundary of the conductor, and used atomistic simulations of thin metallic wires to give realistic atomic configurations, from which we have derived the scattering potential. Just like in the statistical analysis of the experimental results, we consider many different nanowires of different geometries and perform a similar analysis of the simulation results. For the present results for $\mathrm{Au}$ wires, we find that we can reproduce not only the conductance quantization of the experiment, but also the widths of the peaks and their weights. Our analysis suggests that the main reason why the quantization is not destroyed by scattering is that the plastic deformation of the metallic contact works to make the typical wavelength of the scattering potential larger than an atomic diameter and $\lambda_{F}$. However, impurities or other sources of short wavelength scattering may easily destroy the conductance quantization.

In the following, we start in Sec. II by presenting the experimental results and discuss the experimental procedures and the data analysis. In Sec. III, we then describe our molecular dynamics simulations of the atomic structure and the dynamics of nanowires. On that basis, we can discuss the electron transport in the nanowires. We first give the simplest adiabatic theory in Sec. IV, and then go on to discuss the role of scattering from the boundary of the conductor in Sec. V. We conclude in Sec. VI with a summary and discussion of the most important results.

\section{EXPERIMENTAL INVESTIGATIONS OF STM NANOWIRES}

\section{A. Experimental conditions}

All experiments are carried out at room temperature with a compact, fully automated, high stability UHV STM, which offers atomic-resolution images of metal surfaces on a routine basis in the normal constant-current mode. ${ }^{24-26}$ The STM tip is made of a [100]-oriented single crystal W wire, which is electrochemically etched until nice, sharp crystal facets appear. A possible in situ tip cleaning can be performed by ramping the bias voltage $\left(V_{t}\right)$ to $5 \mathrm{~V}$ in $100 \mathrm{~ms}$ or by drawing a high tunnel current $(\sim 10 \mathrm{nA})$ with $V_{t} \sim 1 \mathrm{~V}$. Moreover, the tip can also be reshaped in situ by indenting it into the surface if, for instance, an undesired double tip is present. Apart from the STM, the UHV chamber is equipped with standard facilities for surface cleaning and characterization. The $\mathrm{Au}(110)$ surface used in this study was cleaned by repeated Ne sputtering until perfect, impurity-free surfaces were obtained, as observed by LEED, AES, and STM.

Prior to an experiment, the clean Au surface is scanned in a constant-current mode, and atomic resolution is obtained with typical tunneling parameters $I_{t} \approx 5 \mathrm{nA}$ and $V_{t} \approx 5 \mathrm{mV}$ (see Fig. 1), which correspond to a tunnel resistance of the order of $1 \mathrm{M} \Omega$, or equivalently, a conductance of the order of $1 \mu \mathrm{S}$. The motion of the tip during an indentation process is depicted in Fig. 2, where 


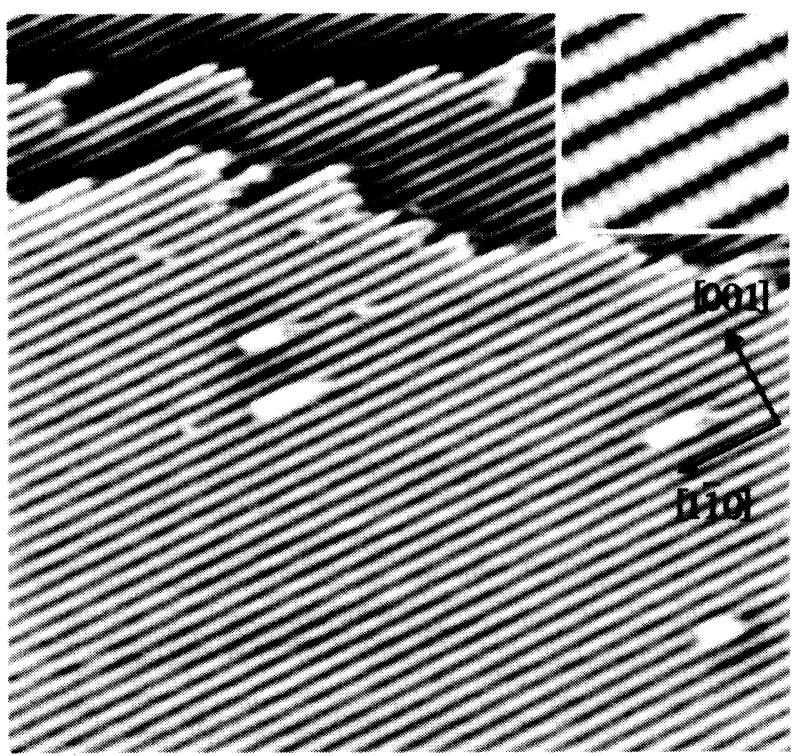

FIG. 1. A $325 \times 335 \AA^{2}$ constant-current image of the clean, $(1 \times 2)$ reconstructed $A u(110)$ surface recorded prior to an indentation with tunneling parameters $I_{t} \approx 4 \mathrm{nA}$ and $V_{t} \approx 3 \mathrm{mV}$. The inset shows a $40 \times 40 \AA^{2}$ closeup of the rows running along the $[1 \overline{1} 0]$ direction.

the origin of the displacement scale corresponds to the initial tunneling position. When the tip passes a preselected point in the normal $x-y$ scan, the feedback loop is opened, and the indentation is performed: The tip is retracted $\approx 15 \AA$ to a position where it is allowed to relax for $\approx 1000 \mu$ s before it is driven towards the surface at a rate of $\approx 500 \AA / \mathrm{s}$. The tip advances beyond the initial tunneling position to an indentation depth of $\approx 3 \AA$, where it is forced to stay for another relaxation time of $\approx 1000 \mu \mathrm{s}$. Subsequently, the tip is withdrawn from the surface, again at a rate of $\approx 500 \AA / \mathrm{s}$, back to the starting point of the indentation and, finally, the tunneling condition is reestablished by a gentle tip approach.

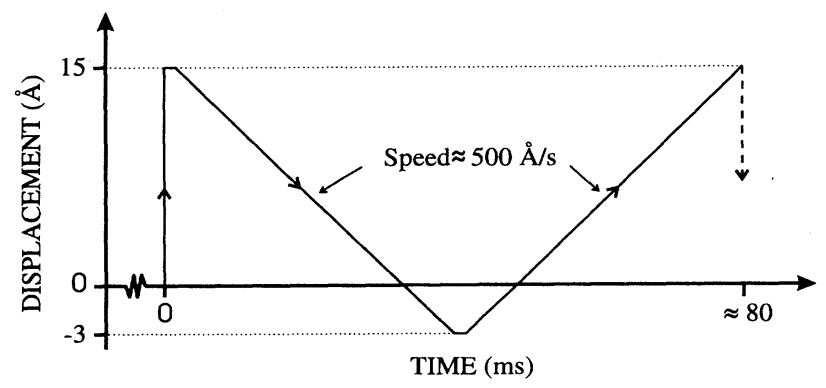

FIG. 2. Depth-time sequence in an indentation. The origin of the displacement scale is given by the tunneling position before the feed back loop is opened, and, therefore, it depends on the tunneling parameters. In the final tip approach, the current is continuously monitored, and the motion is terminated as soon as the previous tunneling conditions are satisfied.

\section{B. Measuring the conductance}

In order to measure the conductance $G_{t}$ (or equivalently the resistance $R_{t}$ ) in the tunnel junction during the indentation process, it is necessary to know both $I_{t}$ and $V_{t}$. From an experimental point of view, an easy way to accomplish this is to keep the bias voltage constant and then measure $I_{t}$. However, one has to worry whether the experimental setup is capable of keeping the real tunnel voltage $V_{t}=I_{t} R_{t}$ equal to the bias voltage $V_{\text {bias }}$ during the indentation. The assertion that $V_{t} \approx V_{\text {bias }}$ is only true when the effective input impedance of the preamplifier, $R_{\text {in }}$ (in our case $11 \mathrm{k} \Omega$ ), is orders of magnitude smaller than $R_{t}$. But this is not the case in an indentation experiment, since we often reach conductances above $1000 \mu \mathrm{S}\left(R_{t} \lesssim 1 \mathrm{k} \Omega\right)$. We circumvent this problem by adding on a low noise $\left(\sim 3 \mu \mathrm{V}_{\mathrm{RMS}}\right)$ high impedance $\left(>1 \mathrm{~T} \Omega\right.$ ) voltage preamplifier which measures $V_{t}$ directly across the STM junction. This setup allows a determination of $G_{t}$ over six orders of magnitude, since $G_{t}$ is determined by the variation of $I_{t}$ in the low conductance regime, whereas it is the variation in the measured $V_{t}$ that determines $G_{t}$ in the high conductance regime.

Even though the conductance measurement has been calibrated against known resistances, there are two further complications in the experiments. Due to small temperature gradients in the UHV chamber the voltage is slightly offset by thermovoltages. By indenting the tip $200 \AA$ into the surface, these thermovoltages have been measured to $11.5 \pm 1.0 \mu \mathrm{V}$, and the conductance measurements have thus been correspondingly corrected. Another problem, which has not been discussed in previous studies of quantized conductance in metals, is the internal resistance in the electrodes, connecting the ballistic system to the macroscopic world. In the macroscopic part of the electrodes, the resistance is negligible, however, because of the small dimensions close to the ballistic system under investigation, the total resistance can be of importance. This serial resistance depends strongly on the actual geometry, and in STM experiments, it can therefore attain any value from less than one ohm to several hundreds of ohms depending on the specific shape of the tip apex. Based on indirect measurements and evaluations, we have estimated this tip resistance to lie in the range $100-200 \Omega$. In the derivation of the conductance we have, thus, taken into account a serial tip resistance of $150 \pm 50 \Omega$. The awareness of such complications is important, although they only introduce a correction of a few percent of a quantum unit in the absolute numbers.

\section{Data analysis}

\section{Current, voltage and conductance}

Figure 3 shows a set of $I_{t}, V_{t}$, and deduced $G_{t}$ data from an indentation measurement on the $\mathrm{Au}(110)$ surface. In the beginning of the indentation sequence, when the tip is very far from the surface, $I_{t} \approx 0$ and $V_{t} \approx V_{\text {bias }}$ (the finite value of $G_{t}$ is due to an offset of a few pA in $\left.I_{t}\right)$, but as the tunneling regime is approached, a rapidly increasing $I_{t}$ is detected [Fig. 3(a)]. Simultaneously $V_{t}$ 

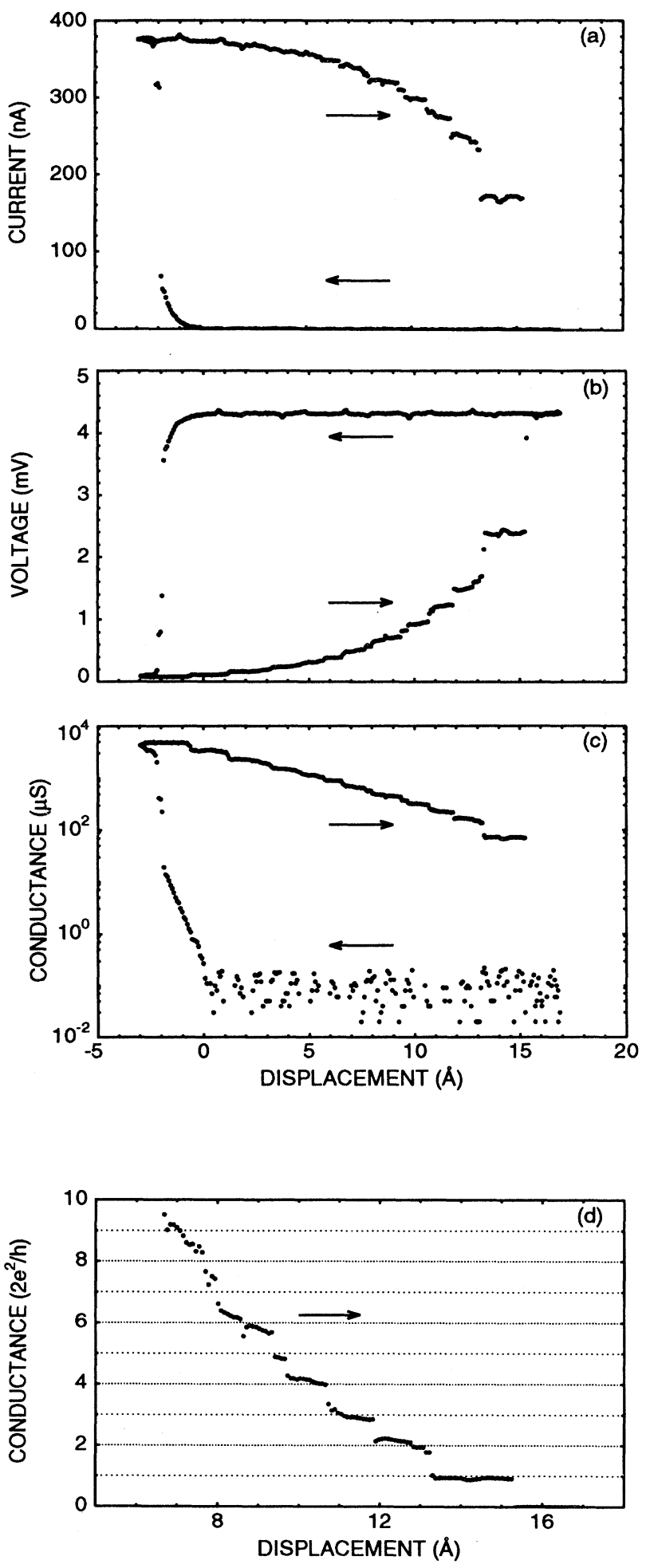

FIG. 3. Simultaneously measured current $I_{t}$ (a) and voltage $V_{t}$ (b) during an indentation. The deduced conductance $G_{t}=I_{t} / V_{t}$ is shown below on a logarithmic scale (c) and on a linear scale (d). The direction of tip motion is given by the arrows, i.e., a left-pointing arrow indicates an approaching tip, while a retracting tip is indicated by a right-pointing arrow. drops to very low values [Fig. 3(b)], showing that $V_{t}$ is indeed not constant even though $V_{\text {bias }}$ is. From Fig. $3(\mathrm{c})$ it is seen that $G_{t}$ increases exponentially in this region, indicating a constant value of the so-called "apparent barrier height." 27,28 This continues all the way up to a point where a sudden increase in $G_{t}$ over several orders of magnitude sets in. During the subsequent retraction of the tip, $G_{t}$ does not follow the characteristic exponential behavior of tunneling, rather it stays at very high values until it suddenly drops back to the level of the in-going curve after a total tip retraction of 10-20 $\AA$.

The jump in $G_{t}$ away from the tunneling regime in the in-going curve is correlated with the remarkable hysteresis seen in Fig. 3, that is, the hysteresis is observed if and only if a jump occurs; otherwise $G_{t}$ is reversible. Thus, we attribute the jump in $G_{t}$ to the formation of a point contact between tip, and sample. ${ }^{10,12}$ Furthermore, since the conductance does not fall off exponentially during the following retreat of the tip, we conclude that the metallic contact remains until the dramatic drop in $G_{t}$ sets in at the end of the outgoing curve. In other words, a connective neck between the tip and the sample is created, stretched $10-20 \AA$, and finally disrupted during the retraction of the tip.

Here, it is very important to note that the tunnel current preamplifier is far from saturation at all times. The almost constant value of $I_{t}$ during elongation of the neck is solely determined by $V_{\mathrm{bias}} / R_{\mathrm{in}}$, since in this region $R_{t}$ is vanishing compared to $R_{\mathrm{in}}$.

\section{Wetting of the tip}

When an experiment is started, the reproducibility of the indentation measurements is fairly low. One indentation to a depth of $10 \AA$ may not lead to the formation of a neck at all, while another indentation to a depth of $2 \AA$ produces a neck with a stretching length of more than $20 \AA$. These long stretching necks are often connected with a considerable tip change and a deposition of material on the crystal over an area of $10^{4} \AA^{2}$.

After a sequence of $\sim 30-100$ initial indentations, each done at a new clean spot of the crystal, the situation has changed markedly. Now a single indentation will result in a very small modification of the tip, i.e., the change in tip length (measured as the change in substrate height in the corresponding topographic image) is less than $0.5 \AA$, and resolution of the [110] rows is maintained. Furthermore, at a fixed depth of indentation, the stretching length of the neck is constant to within $\pm 4 \AA$. We suggest that such a stable tip configuration is a result of natural selection and the fact that our tip is made from a single crystalline wire with a fixed orientation, that is, if an indentation by chance produces an especially stable configuration of the tip, it is much less probable that the tip will undergo a change in the following indentations.

Another likely effect of this initial "cleaning" procedure is a wetting of the tip with substrate atoms, due to the lower surface energy and the higher self-diffusion coefficient for Au compared to W, a picture that is supported by simulations by Landman et al. in the case of 
a Ni tip on a Au substrate. ${ }^{12}$ Because of the wetting, the connective neck will mainly consist of substrate material, and thus we will, in the following, interpret the observed behavior of the conductance as characteristic for a constriction made of $\mathrm{Au}$ atoms.

\section{Quantized conductance}

When the conductance is plotted on a linear scale [Fig. 3(d)], it is seen that during the stretching of the neck, $G_{t}$ is quantized in units of $2 e^{2} / h$, but exactly which quanta are observed differs from one indentation to another. However, it is also obvious from Fig. 3(d) that not all the plateaus are lying exactly on an integer multiplum of $2 e^{2} / h$, but instead there is a tendency for spreading around those values. Moreover, Fig. 3 is not representative for a typical indentation, since, in general, only two or three conductance plateaus are revealed. So in order to investigate to which extent the conductance is indeed quantized in units of $2 e^{2} / h$, we have carried out a detailed statistical analysis, including data points from many indentations.

In such an analysis, it is clearly not desirable to include measurements of $G_{t}$, which do not reflect the nature of the conductance in the neck, but rather show features due to an unstable tip or impurities in the neck. Furthermore, in order to reduce possible complications due to the large opening angles in a point contact ${ }^{23}$ and make the experiment as specific as possible, we want to concentrate on the conductance in an atomic-sized wire. These considerations give rise to the following three criteria which the individual measurements of $G_{t}$ have to fulfill in order to be included in the statistics.

(1) The in-going part of the conductance curve should display an exponential rise in the tunneling regime in correspondence with normal tunneling characteristics [see Fig. 3(c)]. Occasionally, a $G_{t}$ is recorded which exhibits several jumps in this region [see Fig. 4(a)]. This may be associated with a rearrangement of the structure of the tip. If, on the other hand, a pure exponential behavior is detected, the resulting "apparent barrier height" is always fairly high $(\approx 5 \mathrm{eV})$, indicating a clean tip. ${ }^{10}$

(2) When the neck breaks, $G_{t}$ should drop from a level of the order of one quantum unit all the way down to the noise level. If, instead intermediate plateaus are detected [see Fig. 4(b)], then a mechanical contact (a neck) remains to exist despite the very small conductance, which is not in correspondence with a metallic contact. Therefore these plateaus are interpreted as the result of tunneling through an impurity in the neck, in good agreement with the exponential decrease often seen in such plateaus.

(3) During the retraction of the tip, a nanowire with a length of at least $8 \AA$ should form between tip and surface.

Data points from the outgoing part of those $G_{t}$ that satisfy these three criteria are the basis for a continuous histogram, which then will reveal the distribution of data points. The histogram is made in the following way: First, all the measured values of $G$ are sorted in increasing order (see Fig. 5). Second, the histogram value at a
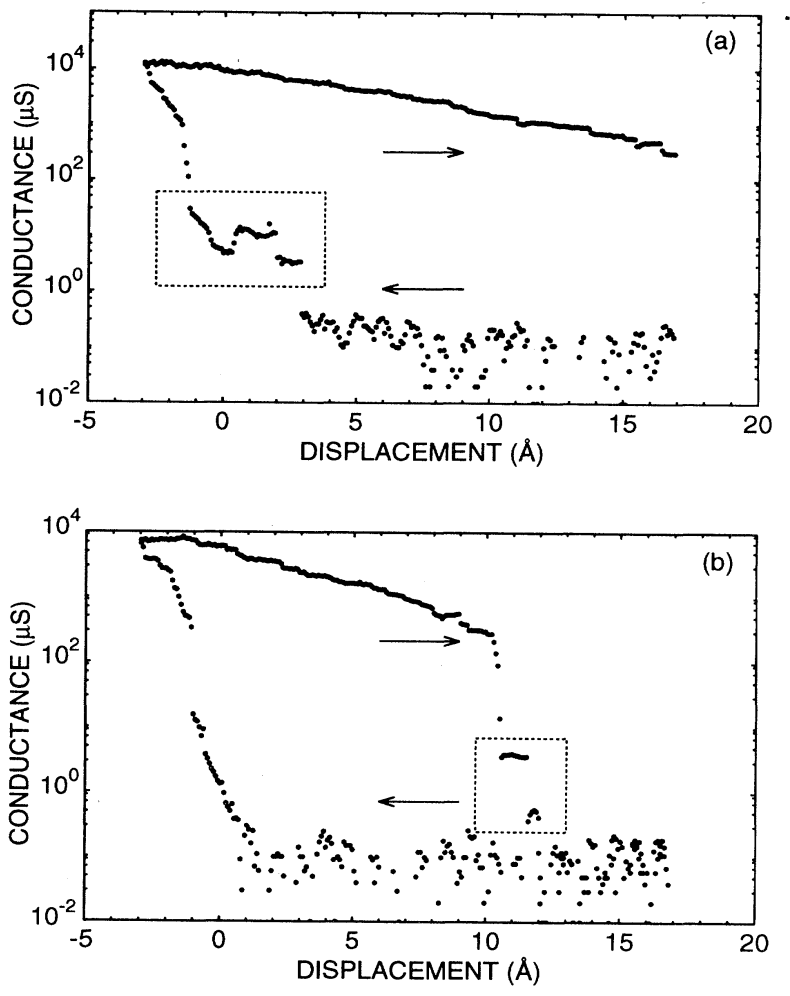

FIG. 4. When $G_{t}$ exhibits jumps in the tunneling region as the tip approaches the surface, it is attributed to an unstable tip (a). Conductance plateaus far below one quantum unit $(\approx 77.5 \mu \mathrm{S})$ in the outgoing part of $G_{t}(\mathrm{~b})$ are not in correspondence with a metallic contact. Instead, they are attributed to tunneling through an impurity in the neck.

certain measured $G_{i+w}$ is calculated as

$$
H\left(G_{i+w}\right)=\frac{\rho_{i+w}}{N}, \text { where } \rho_{i+w}=\frac{2 \omega}{G_{i+2 w}-G_{i}}
$$

is the density in a window of $2 w$ data points, and $N$ is the total number of data points in the histogram. The normalization factor of $1 / N$ ensures that the integrated histogram is $\approx 1$.

In Fig. 6(a), we show such a histogram for $\mathrm{Au}(110)$ based on 6562 data points from 227 indentations fulfilling the criteria above out of a total of 429 different indentations. By fitting the individual peaks to a Lorentz distribution with a linear background, we have found the peak positions to be $1.000 \pm 0.004,2.03 \pm 0.03$, and $2.99 \pm 0.05$, with the corresponding areas and widths shown in Figs. 6(b) and (c). The origin of the stated uncertainties is mainly the uncertainty of the tip resistance described in Sec. II B. From Fig. 6, it is seen that

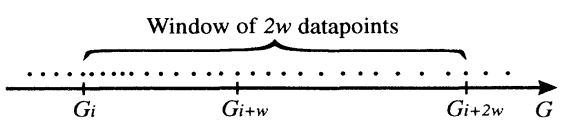

FIG. 5. The histogram value at a conductance of $G_{i+w}$ is calculated from the density of data points in a window of $2 w$ data points. See text for further details. 
the peak at one conductance unit is very sharp and contains nearly a third of all data points in the histogram. Moreover, the peaks at two and three units are easily distinguished from the background level, and the peaks are lying on an integer number, $n$, of quantum units.

We thus conclude that under clean, well controlled conditions, the conductance in a constriction drawn between an STM tip and a metal surface is indeed quantized in units of $2 e^{2} / h$. However, it is seen that the data points are distributed around the integer values, and from a thorough analysis of the histogram, it is revealed that the areas/widths of the peaks decrease/increase with increasing $n$.

The remainder of the paper is devoted to our theoretical modeling of the indentation experiments and a discussion of the experimental findings based on the modeling. In particular, we shall focus on the scaling of the areas and widths of the peaks with $n$.
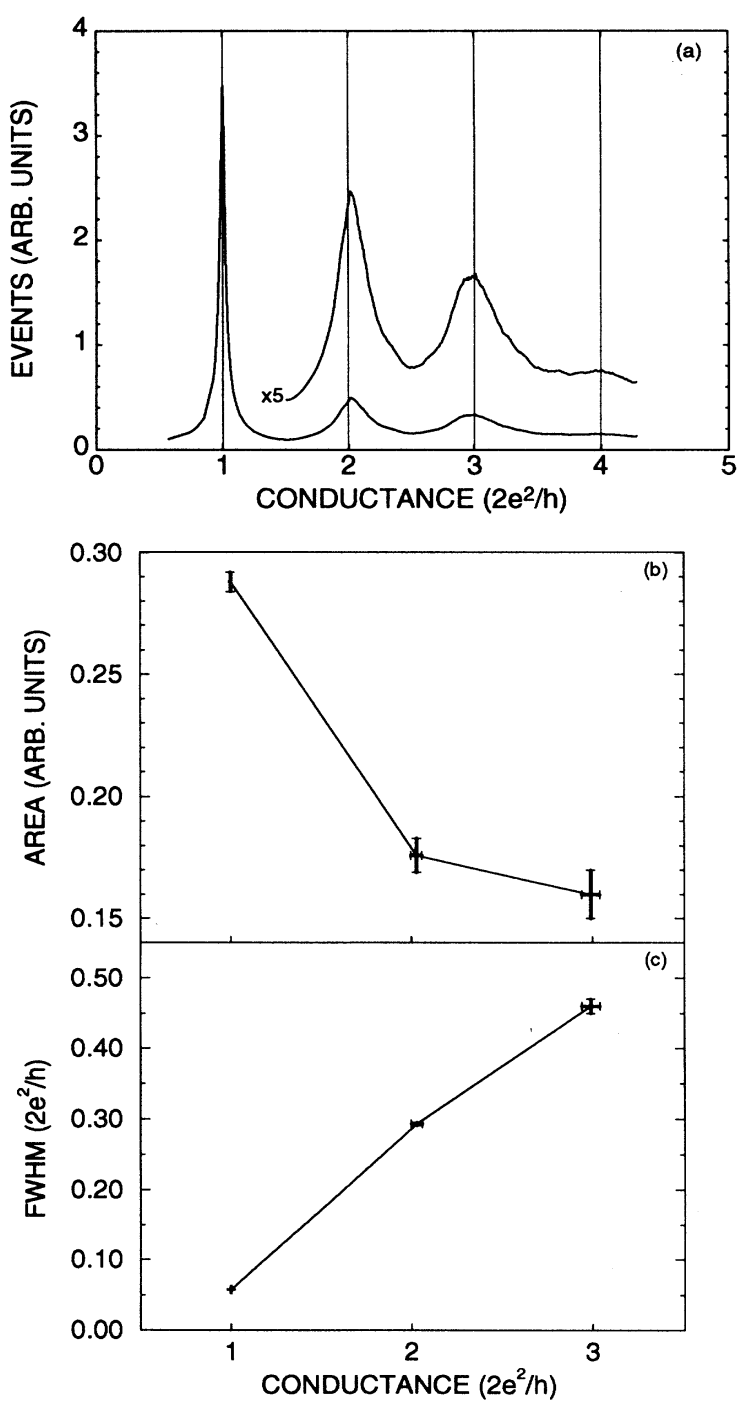

FIG. 6. Continuous conductance histogram based on 6562 data points from 227 indentations on $\mathrm{Au}(110)$ (a). The corresponding areas and widths of the peaks are shown in (b) and (c), respectively.

\section{MOLECULAR DYNAMICS SIMULATIONS OF NANOWIRES}

As a starting point for a theoretical description of the electron transport through point contacts between two metals, we have performed a series of molecular dynamics simulations of the formation of the contact and the elongation process. The molecular dynamics simulations have been performed using an interaction potential derived from the effective-medium theory. ${ }^{29}$ While approximate, this method is known to give a reasonable description of the interatomic interactions in fcc metals. ${ }^{30}$

First, we have studied the formation of the contact. As discussed in the previous section, we assume that the surface metal wets the tip so that the contact is consisting of the surface metal only. In the simulations, a sharp pyramid-shaped tip is brought into contact with a surface, see Fig. 7. At room temperature, diffusion processes will take place on the time scale of the experiment (ms), and this will influence the shape of the contact. However, the time scale of the simulation (ns) is much too short to allow for diffusion to occur. In order to compensate for this, we have enhanced the atomic mobility by annealing the tip to $1050 \mathrm{~K}$ for $270 \mathrm{ps}$. During the annealing, atoms migrate towards the lower substrate, and this leads to the formation of a neck with a structure that is significantly different from the original one.

After the annealing process, the tip is retracted. The temperature of the system is kept at $300 \mathrm{~K}$, by imposing corresponding fluctuating and frictional forces on the atoms in one layer in the bottom of the lower substrate. Figure 7 shows the evolution of the neck during the stretch until it finally breaks. After the tip has broken, an island of material is observed on the surface just like in the experiment, and we note that this "tip-indentation procedure" also gives a fine, sharp tip which will be perfectly suited for atomic-resolution STM.

In Fig. 8, we show how the force on the tip and the cross-sectional area (to be defined in Sec. V) of the neck at the thinnest point evolves during the stretch along with the conductance calculated, using the procedure we discuss in the following sections. It can be seen that the cross-sectional area decreases slowly, while the adhesive force is built up by elastic deformation. This goes on un-

(a )
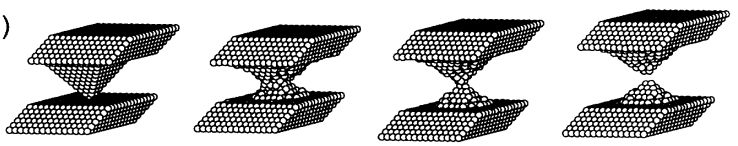

(b)
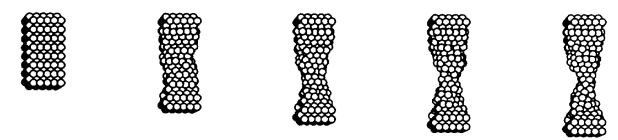

FIG. 7. (a) In this simulation, we see how diffusion processes makes the tip join smoothly to the surface, creating a neck. Subsequent retraction of the tip makes the evolution of the neck closely resemble the stretch of a thin metallic wire. (b) Snapshots from a simulation of the stretch of a nanowire with $5 \times 5 \times 16$ structure along the [100] direction. 


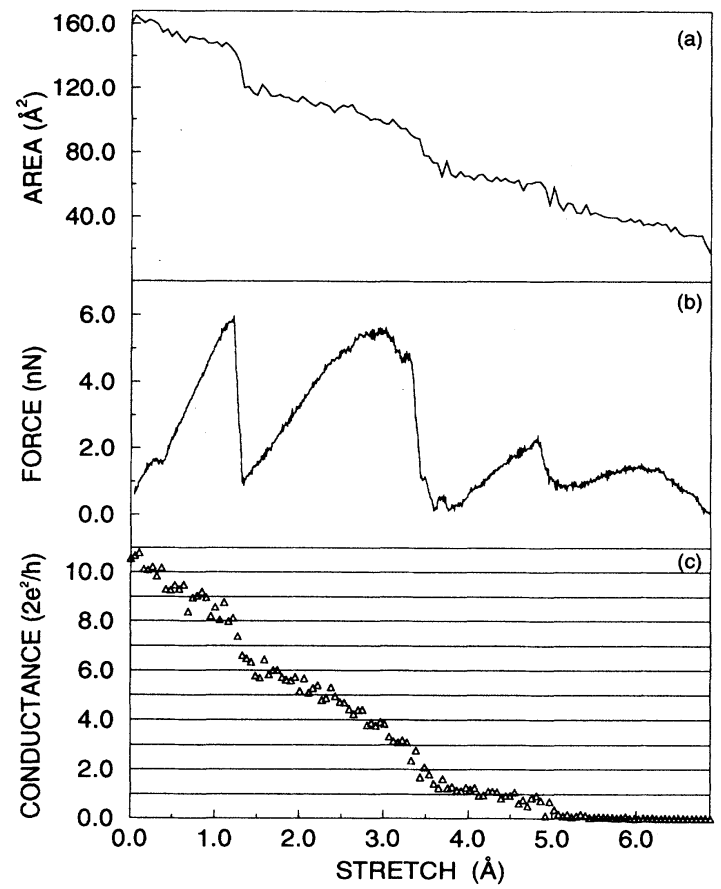

FIG. 8. Typical evolutions of the area with stretch (a). It is seen that the area decreases rather linearly disrupted by sudden jumps corresponding to major atomic rearrangements in the neck. This is reflected in the correlation with jumps in the force on the tip showing buildup and subsequent release (b). In (c) the corresponding conductance curve is shown.

til a sudden restructuring of the neck occurs. During the restructuring, the cross-sectional area and conductance change along with a drop in the adhesive force. This is in agreement with the molecular dynamics simulations by Landman et al. ${ }^{12}$ Furthermore, we note that the jumps in force are around $5 \mathrm{nN}$, except for the thinnest necks, which is in agreement with the magnitude of the force jumps measured experimentally by Stalder and Dürig. ${ }^{19}$ On the other hand, their experiment indicates that a conductance plateau corresponds to a slow decrease in the adhesive force, and the change in conductance is correlated to a steep increase in the adhesive force. The experimental regime for force measurements by Dürig and Stalder stops at about six conductance quanta, so a direct comparison between theory and experiment is not possible.

It can be seen from Fig. 7 that the central part of the neck can be viewed as a thin metallic wire with a constriction. We can utilize this by replacing the "real" neck in Fig. 7(a) by the nanowire shown in Fig. 7(b). During stretch, the wire develops a constriction just like the neck, and we find that the area versus stretch functions for the nanowires are very similar to those for the "real" neck for constrictions smaller than about five atoms. For simplicity, we have thus used nanowires in the following.

In order to make a statistical analysis of the "computer experiments" in analogy with the treatment of the experimental results given above, we have performed room-temperature Langevin dynamics simulations of the stretch of 12 different Au wires. Starting configurations with 8-25 atoms in cross section and a length of 16 atomic layers arranged in one of three different structures ([100], [110], or [111] along the direction of the wire) with varying ratio of side lengths have been chosen. These simulations are used as input into the calculation of the electron transport, i.e., the conductivity, which we shall discuss in the following.

\section{QUANTIZED CONDUCTANCE IN ONE-DIMENSIONAL NANOWIRES}

We assume that transport through the neck structure can be described by a coherent propagation of the electrons. This means that all length scales characterizing destruction of this coherence, ${ }^{2,31,40}$ e.g., the mean free path of the electron $(l)$, are assumed to be larger than the dimensions of the neck structure.

Let us first consider a one-dimensional conductor connecting two-electron reservoirs. In both reservoirs, the electrons are in thermodynamical equilibrium, and chemical potentials $\left(\mu_{1}\right.$ and $\left.\mu_{2}\right)$ can be assigned to each reservoir. We assume that the reservoirs completely randomize the phase of an electron entering from the conductor, so there will be no interference effects between the part of the electronic wave function traveling from the conductor to the reservoir and the part entering the conductor from the reservoir. We, furthermore, assume that there is a perfect matching between the conductor and the reservoirs: No electrons coming from the conductor are reflected at the point where the conductor joins the reservoir. The conductor is perfect, except for a single barrier with transmission coefficient $T$.

Under these assumptions, the conductance is given by the Landauer-Büttiker formalism. ${ }^{32,33}$ The current from reservoir 1 to reservoir 2 is carried by electrons with energies between their respective chemical potentials $\mu_{1}$ and $\mu_{2}$ and a wave vector in the direction opposite to the current:

$$
I=e v_{F} \frac{g\left(E_{F}\right)}{2}\left(\mu_{1}-\mu_{2}\right) T
$$

where $e$ is the electron charge, $v_{F}$ is the electron velocity (at the Fermi energy), and $g\left(E_{F}\right)$ is the density of states at the Fermi level for the one-dimensional conductor. The number of electrons contributing to the current is thus given by $\frac{g\left(E_{F}\right)}{2}\left(\mu_{1}-\mu_{2}\right)$. Since the voltage between the reservoirs is given by the difference in chemical potential, $e V=\mu_{1}-\mu_{2}$, the conductance $G$ is

$$
G=e^{2} v_{F} g\left(E_{F}\right) T=\frac{2 e^{2}}{h} T
$$

where it has been used that the density of states at the Fermi energy for a one-dimensional electron gas is $g\left(E_{F}\right)=2 /\left(\pi \hbar v_{F}\right)$, including the spin degeneracy.

It is seen that if the transmission is perfect, the conductance is $2 e^{2} / h$, the size of the quantum observed in the experiments. If many independent conduction channels are present, the total conductance becomes ${ }^{33}$ 


$$
G=\frac{2 e^{2}}{h} \sum_{i} T_{i}
$$

where $T_{i}$ is the probability that an electron entering channel $i$ is transmitted in any channel. Different conductance channels could be present if the electrons can be labeled with quantum numbers not directly related to the motion along the wire, and if the electrons with different quantum numbers can be treated independently. In such a case, the current in Eq. (2) will flow in each channel.

These equations are strictly speaking only valid at zero temperature; at finite temperatures, Eq. (4) becomes

$$
G=\frac{2 e^{2}}{h} \int_{-\infty}^{\infty} \sum_{i} T_{i}(E)\left(-\frac{d n_{F}}{d E}(E)\right) d E
$$

where $n_{F}$ is the Fermi function. However, the smearing of $\sum_{i} T_{i}(E)$ by the last factor is only important if the transmissions vary with $E$ on a scale of the order of $k T$. This effect is not important in this context, as will be shown later.

To show how this one-dimensional analysis is relevant for a real three-dimensional nanowire, it is necessary to take a closer look at the wave functions of the conducting electrons: We assume that the electron motion is free-electron like and use a coordinate system with the $z$ axis in the direction of the wire. If the $x, y$ behavior of the wave function varies sufficiently slowly with $z$, we can separate the problem into a series of isolated one-dimensional propagations in $z$, defining the conductance channels. To be more precise, we can for each value of the $z$ coordinate formally solve the twodimensional Schrödinger equation in $x$ and $y$ and expand the three-dimensional wave function $\psi$, using these twodimensional wave functions $\chi$ as a basis:

$$
\psi(x, y, z)=\sum_{n} \chi_{z, n}(x, y) \phi_{n}(z)
$$

where $\chi_{z, n}$ are the solutions to

$$
\begin{array}{r}
{\left[-\frac{\hbar^{2}}{2 m}\left(\frac{\partial^{2}}{\partial x^{2}}+\frac{\partial^{2}}{\partial y^{2}}\right)+V\left(x, y, z_{0}\right)\right] \chi_{z_{0}, n}(x, y)} \\
=E_{n}^{\perp}\left(z_{0}\right) \chi_{z_{0}, n}(x, y),
\end{array}
$$

and $\phi_{n}$ are the $z$ dependent expansion coefficients.

When this expansion is inserted in the threedimensional Schrödinger equation, we get

$$
\begin{array}{r}
\left(-\frac{\hbar^{2}}{2 m} \frac{\partial^{2}}{\partial z^{2}}+E_{n}^{\perp}(z)\right) \phi_{n}(z)+\sum_{m} \hat{F}_{n m}^{\text {coupling }} \phi_{m}(z) \\
=E_{F} \phi_{n}(z),
\end{array}
$$

where the electron energy is set to $E_{F}$, since only electrons in a narrow region around that energy contribute to the current. The operators $\hat{F}_{n m}^{\text {coupling }}$ couple the different $z$ solutions $\phi_{n}$. They have the form

$$
\begin{aligned}
\hat{F}_{n m}^{\text {coupling }}= & -\frac{\hbar^{2}}{2 m}\left(\left\langle\chi_{n}(z) \mid \frac{\partial^{2}}{\partial z^{2}} \chi_{m}(z)\right\rangle\right. \\
& \left.+2\left\langle\chi_{n}(z) \mid \frac{\partial}{\partial z} \chi_{m}(z)\right\rangle \frac{\partial}{\partial z}\right) .
\end{aligned}
$$

This gives the motivation for the particular choice of expansion: If the variation of $\chi_{n}$ with $z$ in Eq. (6) is sufficiently slow - the adiabatic case - the coupling between equations with different $n$ by $\hat{F}_{n m}^{\text {coupling }}$ can be neglected, and we are left with an isolated equation for each $n$, which has the form of a one-dimensional Schrödinger equation in $z$ with an effective potential barrier $E_{n}^{\perp}(z)$. Equation (7) defines $\chi_{z, n}$ uniquely apart from a phase factor that should be chosen such that $\left|\frac{\partial}{\partial z} \chi_{z, n}\right|$ is minimized and

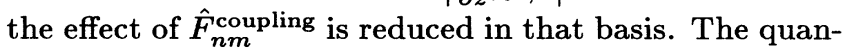
tum numbers $n$, which describe the motion perpendicular to the wire, therefore define the individual conductance channels in the Landauer-Büttiker formalism of Eq. (4).

Let us now be more specific and discuss the potential in which the independent electrons move. First of all, we note that, although bulk-Au has $d$ bands, the transport properties are dominated by freelike carriers. This is reflected in the shape of the Fermi surface, which can be described well by the free-electron sphere with some minor bulge outs in the $\langle 111\rangle$ directions. The band mass determined from the density of states at $E_{F}$ is close to one $\left(m_{\text {band }} / m=1.09\right.$ from Ref. 34$)$. This shows that the ion potential for $\mathrm{Au}$ does not scatter the electrons at the Fermi surface considerably. It is thus reasonable for a semiquantitative discussion of the possibilities of conductance quantization to neglect the scattering inside the nanowire due to the single ions and employ a free-electron picture.

We, therefore, assume that the electrons move in a flat potential confined by a hard wall, and we furthermore assume that the cross section of the conductor for each $z$ is a rectangle with side lengths $L_{x}(z)$ and $L_{y}(z)$. Then we can calculate $E_{n}^{\perp}(z)$ and $\chi_{z, n}(x, y)$ analytically:

$$
\begin{aligned}
E_{n}^{\perp}(z)= & \frac{\pi^{2} \hbar^{2}}{2 m}\left(\frac{n_{x}^{2}}{L_{x}(z)^{2}}+\frac{n_{y}^{2}}{L_{y}(z)^{2}}\right), \\
\chi_{z, n}(x, y)= & \frac{2}{\sqrt{L_{x}(z) L_{y}(z)}} \sin \left(\frac{n_{x} \pi}{L_{x}(z)} x-\frac{n_{x} \pi}{2}\right) \\
& \times \sin \left(\frac{n_{y} \pi}{L_{y}(z)} y-\frac{n_{y} \pi}{2}\right) .
\end{aligned}
$$

A similar behavior will be seen with other shapes of the confining potential, i.e., $E_{n}^{\perp}$ increases with increasing quantum numbers and with decreasing width of the conductor in a way very similar to Eq. (10). The conditions for adiabaticity now amounts to $L_{x, y}$ changing sufficiently slowly with $z$. If we neglect the coupling between channels (we discuss the coupling in the next section), each channel is described by an ordinary Schrödinger equation with $E_{n}^{\perp}$ as a barrier [Eq. (8)]. It is clear that in the simplest case, when the $z$ variation is so slow that there is no tunneling through the barrier and no reflec- 

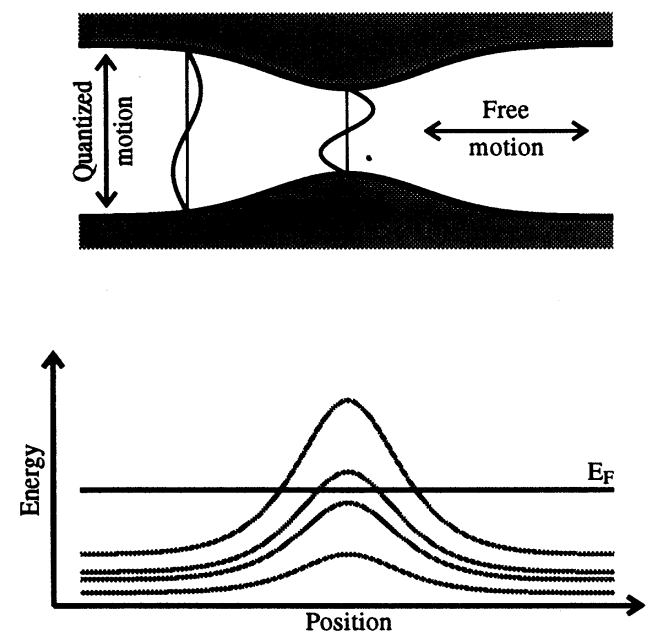

FIG. 9. The quantization of conductance can be explained by the quantization in the transverse direction of a smooth constriction. To each transverse mode corresponds a potential barrier in the longitudinal direction, due to the energy taken up by the transverse motion. The finite number of modes with a barrier less than $E_{F}$ contributes each with the conductance quantum $2 e^{2} / h$.

tion above it, we can assume that a channel $n$ is open (i.e., $T_{n}=1$ ) if the top of the barrier $E_{n}^{\perp}(z)$ is below $E_{F}$ and closed $\left(T_{n}=0\right)$ otherwise. This gives the first crude approximation to the experiment: As the wire is pulled, it becomes more and more narrow, the barriers are increasing, and one by one they pass through the Fermi level and close the corresponding channel. Each time a channel is closed, the conductance drops by $2 e^{2} / h$. This is illustrated in Fig. 9. It should be noted that these steps are only seen if the energy difference between the potential curves in Fig. 9 is large compared to $k T$, i.e., if Eq. (4) is a good approximation to Eq. (5). For an atomic-sized constriction, this difference is of the order of $1 \mathrm{eV}$ and thus far larger than $k T$.

\section{ELECTRON TRANSPORT THEORY INCLUDING SCATTERING FROM THE BOUNDARY}

In this section, we elaborate on the adiabatic, freeelectron model described above in two respects: We include the scattering due to the boundary and we give a better description of the electron potential along the wire. In our description of the electron potential, we maintain the "box-model" description of the electron motion in the $x-y$ plane perpendicular to the wire and take the cross section everywhere to be rectangular with side lengths $L_{x}(z)$ and $L_{y}(z)$. But we include a realistic description of the shape of the hard-wall boundary of the wire and include the variation of the average potential along the wire. This is done in the following way: For a given configuration of the wire, we construct the oneelectron potential by overlapping atomic electron densities and calculate the effective one-electron potential in the local density approximation (LDA). The electron densities are taken from self-consistent LDA calculations for the atoms, and we have checked that this procedure gives an adequate description of the absolute values of the potential, as well as the corrugation at the surface by comparing to self-consistent calculations for a $\mathrm{Cu}(111)$ surface. ${ }^{35}$ We have taken the atomic electrostatic potentials and densities to be constant inside a given radius in the core region, corresponding to the core region in a pseudopotential. A cutoff radius for $\mathrm{Au}$, based on a pseudopotential of the Heine-Abarenkov type, of $1.4 \AA$ is used. ${ }^{36}$ Given the potential, we define the "hard walls" to be located at the classical turning points, that is, where the one-electron potential is equal to the Fermi energy of $\mathrm{Au}(\approx 5.5 \mathrm{eV})$.

When the hard-wall boundary is defined, we can determine the area of the free-electron cross section along the wire. We point out that the cross-sectional area obtained in this way does not essentially change the results from the more simple approach used in Ref. 18, where the area is determined by cutting through the Wigner-Seitz spheres placed at the atomic positions, but we obtain a somewhat smoother area function $A(z)$. Figure 10 illustrates how this procedure works. It shows both the atomic configuration, the one-electron potential, and the cross-sectional area along the wire. In this way, we define the effective potential due to the constriction along the wire,

$$
E_{n}^{\perp}(z)=\frac{\hbar^{2} \pi^{2}}{2 m A(z)} \tilde{n}^{2},
$$

where $A(z)$ is the cross-sectional area of the wire, and we have defined

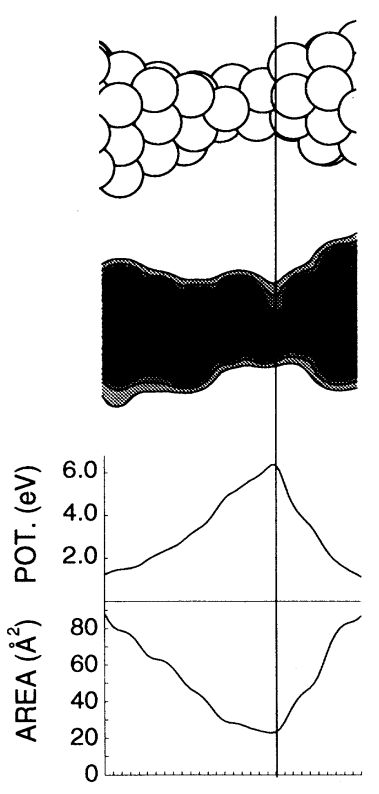

FIG. 10. A cut through a wire and the corresponding potential contour. The last contour is the classical turning point. The derived area and potential $\left[\left(n_{x}, n_{y}\right)=(1,1)\right]$ are shown. 


$$
\tilde{n}^{2}=\alpha(z) n_{x}^{2}+\alpha(z)^{-1} n_{y}^{2},
$$

where $\alpha$ is reflecting the eccentricity and defined as

$$
\alpha(z)=\frac{L_{y}(z)}{L_{x}(z)} .
$$

$A(z)$, as well as $\alpha(z)$, are extracted from the simulation. Of course, enforcing a box geometry is a crude approximation to the real cross section of the wire. Since $\alpha$ is not very well defined, each simulation is followed by conductance calculations with $\alpha, \alpha \pm 0.05$, and $\alpha \pm 0.1$, which we include in our final statistics. In this way, we weaken the dependence on the details of the definition of $\alpha$. In a "soft-wall" model, we would obtain an equivalent model, with $L_{x}$ and $L_{y}$ then being the effective widths of the wave function, including penetration beyond the classical turning points.

As discussed in the preceding section, it is easy in this picture to understand the conductance curve obtained during the stretch of the neck. As the neck is being stretched, the smallest cross-sectional area decreases, and the maxima of the potentials for the channels $\left(n_{x}, n_{y}\right)$ increase, and thus "close" the channels successively, yielding the corresponding jumps in the conductance curve. This is illustrated in Fig. 11, using constriction geometries from one of the simulations. We have shown the effective potentials for the different modes at a certain point in time during the stretching [Fig. 11(a)]. It is seen that these are not just the lowest effective potential multiplied by a constant, because $\alpha$ depends on the position along the wire. We have also shown how the effective potential corresponding to the lowest transverse mode grows during the stretching [Fig. 11(b)].

In the semiclassical limit of large area, $A k_{F}^{2} \gg 1$, we can approximate the number of open channels, $N$,

$$
N \approx \frac{\pi}{4} \tilde{n}_{\max }^{2}
$$

where $\tilde{n}_{\max }$ is the maximum value for which $E_{n}^{\perp}(z) \leq$ $E_{F}$ and we obtain the semiclassical Sharvin ${ }^{1}$ conductance expression using (12),

$$
G_{\text {Sharvin }}=\frac{2 e^{2}}{h} N=\frac{2 e^{2}}{h} \frac{k_{F}^{2}}{4 \pi} A
$$

When the dimensions are of the order $\lambda_{F}$, the number of open channels is better described by including a perimeter correction,

$$
N \approx \frac{k_{F}^{2}}{4 \pi} A-\frac{k_{F}}{4 \pi} P,
$$

where $P$ is the perimeter of the neck as discussed by Torres et al. ${ }^{23}$

It is the eccentricity that determines which steps we are able to observe because of degeneracy or near degeneracy of the potentials for different $\left(n_{x}, n_{y}\right)$. For instance, if $\alpha=1$, only conductance steps at $1,3,4,6 \ldots$ are possible, and for $\alpha \approx 0.6$, steps at $1,2,4,5 \ldots$ are possible. This degeneracy is illustrated in Fig. 12, where we have plotted $\tilde{n}$, the factor determining the degeneracy, versus $\alpha$,
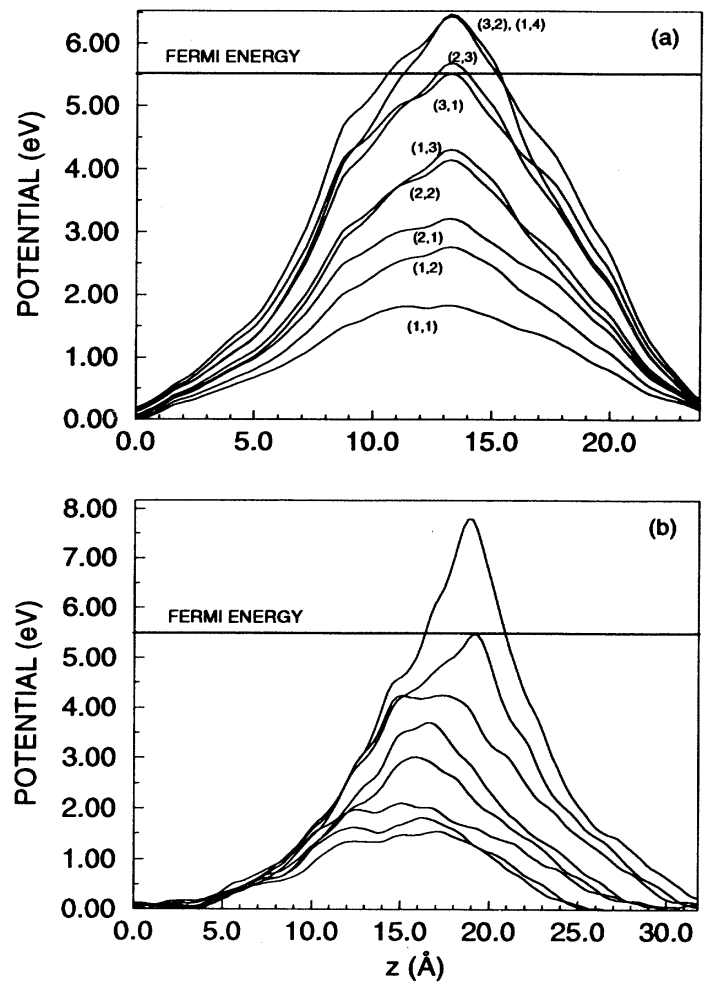

FIG. 11. An example of potentials for different $n_{x}$ and $n_{y}$ along the wire ( $z$ direction) is shown at a particular time during the stretch (a). Tunneling and reflectance above the barrier is present for $\left(n_{x}, n_{y}\right)=(3,2),(1,4)$ and $\left(n_{x}, n_{y}\right)=(3,1),(2,3)$, respectively, whereas the transmission is either very close to 1 or 0 for the rest. Successive $n_{x}=n_{y}=1$ potential curves during stretch are shown in (b). The potential includes both the effect of change in shape and mean cross-sectional potential.

describing the eccentricity. For those values of $\alpha$, where two values of $\tilde{n}$ are close, the barriers of the corresponding modes are almost equal. This in turn means that the conductance channels for these modes will close at the same time giving rise to a jump in the conductance of two units.

Torres et al. $^{23}$ have in their free-electron model assumed the constriction to have a circular transverse cross section and a parabolic-shaped longitudinal cross section with a hard-wall potential. Because of this geometry, the transmission probabilities can be solved exactly, including the infinite free-electron reservoirs. But on the other hand, the axial symmetry imposes a degeneracy so that, e.g., steps at $G=2$ cannot be observed. In our approach, we have access to realistic atomic configurations and, therefore, it is natural to choose a model where the eccentricity easily can be included. Within our model, we can get a rough idea about the shape of the constriction from the observed steps and Fig. 12. For instance, if we see a well-resolved step at $G=2$, the constriction is bound to be eccentric to a certain extent. However, we 


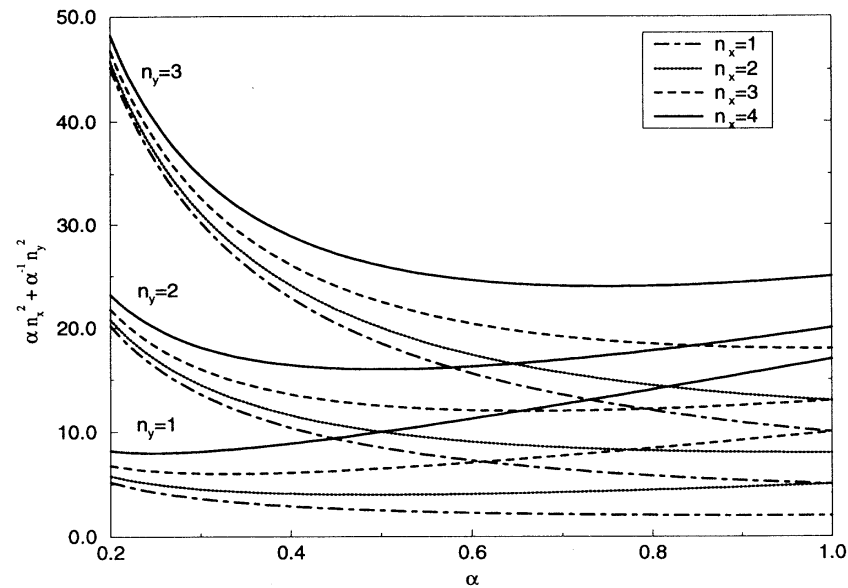

FIG. 12. Plot of $\tilde{n}$ for $n_{y}=\{1,2,3\}$ and $n_{x}=\{1,2,3,4\}$ as a function of $\alpha$. For a given $\alpha$, the distance between neighboring curves determines how well the corresponding steps can be resolved.

do not include a description of the coupling to the infinite reservoirs, which is a complicated matter to describe realistically.

Up until now, we have neglected the effect of a nonconstant potential inside the wire. During the stretching of the wire, the mean potential in a cross section of the wire grows, because there are fewer atoms present in the cross section, and thus there is a relatively larger surface part of the potential. We have included this effect by adding to $E_{n}^{\perp}(z)$ the change in cross-sectional mean of the potential along the wire (zero chosen at the entrance/exit of the wire). The corrugation of this potential due to the single $\mathrm{Au}$ ion is averaged out by taking a mean over several interatomic distances. The reason for this is that the scattering due to the single $\mathrm{Au}$ ion can be neglected, as argued previously. The net effect of the extra contribution to the potential is to close the last channel in the last part of the stretching process somewhat earlier than would be the case using only $E_{n}^{\perp}(z)$. The evolution of the full $(1,1)$ potential with stretch in one of the simulations is shown in Fig. 11(b).

The relevance of the adiabatic approach is not at all obvious in this case where the potential is corrugated on the scale of $\lambda_{F}$. In the following section, we will elaborate further on this point to substantiate that the adiabatic approach can, in fact, be used.

\section{A. Scattering}

Within the free-electron model of our system, we now consider the scattering from the effective potential barrier due to the constriction. As can be seen from Fig. 11, the topmost part of the barrier is nearly a parabola in the last part of the stretching process, with corresponding conduction quanta less than about five. As a first estimate of the transmission, we thus consider the scattering from a single parabola-shaped potential along the direction of the neck $(z)$,

$$
V(z)=V_{\max }-\frac{1}{2} K z^{2} .
$$

For a parabola barrier, an exact expression for the transmission and reflectance above and within the barrier can be found, ${ }^{37}$

$$
T=\frac{1}{1+e^{-2 \pi \epsilon}}, \quad \epsilon=\frac{\sqrt{m}}{\hbar} \frac{E_{F}-V_{\max }}{\sqrt{K}} .
$$

In the tunneling regime, this can be rewritten using the corresponding WKB expression for the transmission $T_{\mathrm{WKB}}$ as

$$
T=\frac{1}{1+T_{\mathrm{WKB}}^{-1}}
$$

where

$$
T_{\mathrm{WKB}}=\exp \left(-2 \int_{V(z)>E_{F}} \sqrt{\frac{2 m}{\hbar^{2}}\left[V(z)-E_{F}\right]} d z\right)
$$

This holds for all $V(z)$ with $V_{\max }>E_{F}$ in the parabola case. For the real potential, Eq. (21) provides a smooth interpolation for $T$ between the regime, where WKB is applicable, $V_{\max } \gg E_{F}$ so $T_{\mathrm{WKB}} \ll 1$, and the regime where a parabola fit to the topmost part of the potential is a good estimate, $V_{\max } \approx E_{F}\left(V_{\max }>E_{F}\right)$. With this approach, we will overestimate the tunneling slightly when the potential cuts $E_{F}$ around "shoulders," where we underestimate the increased reflection, because of the widening of the barrier.

For $V(z)$ with $V_{\max }<E_{F}$, we cannot directly calculate the transmission for the general effective potential. Here, we use the fact that for the parabola-shaped potential $T$ can be written as $T=1-T_{>}$, where $T_{>}$is given by Eq. (19) with $E_{F}$ exchanged by $V_{\max }-\left(E_{F}-V_{\max }\right)$. Therefore, we estimate the transmission $T$ for $V_{\max }<$ $E_{F}$ for the effective potentials by $1-T_{>}$, where $T_{>}$is the tunneling amplitude corresponding to $V_{\max }>\tilde{E_{F}}$ estimated by Eqs. (20) and (21), with $E_{F}$ exchanged by $\tilde{E_{F}}=2 V_{\max }-E_{F}$.

The same type of formula as Eq. (20) was used in the two-dimensional case by Glazman et al. ${ }^{38}$ to express the shape of a step. In the $3 \mathrm{DEG}$ case with a rectangular cross section and a parabolic-shaped potential, we obtain correspondingly the "smearing" $\delta G$ of the conductance quantum step $N$,

$$
\begin{aligned}
\delta G(\gamma) & =\frac{2 e^{2}}{h}\left[1+\exp \left(-\gamma 2 \pi^{2} \sqrt{\frac{2 R}{A^{\frac{3}{2}} k_{F}^{2}}}\right)\right]^{-1} \\
\gamma & =\frac{k_{F}^{2} A}{4 \pi}-N
\end{aligned}
$$

Here, $R$ is the radius of curvature of the constriction. In the general case, Eq. (20) leads to the same shape with an effective radius of curvature, $R_{\text {eff }}$. This radius of curvature will depend on where the Fermi level crosses 
the potential, and, therefore, it depends on the transverse mode we consider. The corresponding width of the transition region between two steps is thus given by

$$
\Delta \sim \frac{1}{2 \pi^{2}} \frac{A^{0.75} k_{F}}{\sqrt{2 R_{\text {eff }}}} .
$$

So, like Glazman et al. ${ }^{38}$ we have found that the leading correction to the reflections within each channel is exponentially small in the "smoothness parameter" $2 \pi^{2} \sqrt{2 R_{\text {eff }}} /\left(A^{0.75} k_{F}\right)$. We could have included perimeter corrections to $N$ in the above formulas, but that does not change the expression for the width. In the $2 \mathrm{D}$ case, it has been shown ${ }^{39,40}$ that the leading corrections to the interchannel reflections are exponentially small in, basically, the same "smoothness parameter" as the withinchannel reflections. This is seen intuitively, since the intrachannel scattering is determined by the curvature of the top of the potential, which depends on how rapidly $L_{x, y}$ changes with $z$, which again is the same factor determining the mixing of channels. Thus, it is (self-) consistent to neglect the effect of interchannel scattering once we have observed that the intrachannel scattering does not ruin the quantum steps, and, therefore, it is reasonable to estimate the total effect of scattering by the within-channel part as done above. Another point concerning the neglect of interchannel mixing is that only mixing that causes reflections back through the constriction will harm the quantization. This is because mixing of the forward moving channels will not change the total transmission and thus the conductance. ${ }^{5}$ In a recent paper, Maaø et al. ${ }^{41}$ show that the neglect of $F^{\text {coupling }}$ in the two-dimensional case, in fact, approximates the exact numerically calculated conductance remarkably well, in spite of strong mode mixing. They find, furthermore, that neglecting $F^{\text {coupling }}$ leads to an underestimate of the degree of conductance quantization.

An upper limit of the scattering can be estimated by taking $R_{\text {eff }}$ to be the Wigner-Seitz radius of $\mathrm{Au}$, $r_{s} \approx 1.6 \AA$. Inserting this and using $E_{F} \approx 5.5 \mathrm{eV}$, we get the width $\Delta \approx 0.16 A^{0.75}$, with $A$ in units of $\pi R_{\text {eff }}^{2}$. Taking the first step to appear at $A=3$, we get $\Delta \approx 0.37$, so we have that all the steps are just being washed out. However, except right where a potential barrier crosses the Fermi energy, the effective radius of curvature is several times larger than $r_{s}$ (see Fig. 11), and the steps appear.

Inelastic scattering is neglected in the above approach. In the experiments, which are done at room temperature, we expect the phonons to be the dominant source of inelastic scattering. Maxwell ${ }^{42}$ calculated the classical conductance through a circular hole with radius $a$ connecting two metals in the limit, where Ohms law is applicable,

$$
G_{\text {Maxwell }}=2 a \sigma,
$$

where $\sigma$ is the conductivity. This is a diffusive limit where the corresponding ballistic limit is given by the Sharvin result (16). A simple interpolation formula between these limits has been found ${ }^{43}$ using the Boltzmann equation,

$$
G=G_{\text {Sharvin }}\left[1+\frac{3 \pi}{8} \Gamma\left(\frac{l}{a}\right) \frac{a}{l}\right]^{-1},
$$

where $\Gamma$ is a slowly varying function with $\Gamma(0)=1$ and $\Gamma(\infty)=0.694$. Thus, the simplest estimate on the effect of inelastic scattering in the quantum limit would be to multiply the conductance by a factor

$$
\frac{l}{l+\sqrt{A}}
$$

which in turn moves the quantum steps towards lower values. A similar interpolation formula has been used by Stalder and Dürig. ${ }^{19}$ This indicates that scattering becomes of increasing importance as the size of the neck structure increases and thus tends to destroy the higher quantum steps. Since the scattering is proportional to the number of states into which an electron can be scattered, i.e., to the density of states, we expect in general $l$ to increase with decreasing neck size, decreasing the inelastic scattering further at small neck sizes.

\section{B. Results of simulation}

Before turning to the results of our simulation, let us just summarize our procedure for calculating the conductance. First, we add approximate electron densities and electrostatic potentials to obtain the total potential for the whole wire. Using this, we calculate the classical turning points for electrons at the Fermi energy. From the turning points, we obtain the area and shape (eccentricity) of the cross sections along the wire, which directly leads us to the effective one-dimensional barrier $E_{n}^{\perp}$ seen by the electrons carrying the current. We add to this the mean potential shift inside the wire. The conductance is now obtained from the transmission of electrons through the effective barrier $E_{n}^{\perp}$, using the Landauer formula. This is done in each time step of the molecular dynamics simulation of the stretching process of the wires, and we obtain the conductance curves with conductance vs stretch for different initial wire structures.

The conductance curves in Fig. 8 and in Figs. 13 (a), (b) are produced using this approach. The curves show features quite similar to the experiment. We see that the curves "slip through" the higher conductances, until they develop plateaus during the last part of the stretch. These are seen mainly at $G=1,2$, and 3 . Often jumps from 3 directly to 1 , or only plateaus at 1 or 1 and 2 are seen, like in the experiment. This is in our simulation controlled by the evolution of the eccentricity along with the area. We have observed a simulation [Fig. 13(b)], where the scattering to a very high extent smeared out the transition between the $G=1$ and $G=2$ step and resulted in a step at a noninteger value between $G=3$ and 4. This structure appears because of a stable neck configuration for a considerable time during the stretch where the barrier results in high scattering. Such features are also seen in the experiment. In general, we see in both experiment and simulation that the higher quantum steps are "washed out." In fact, in our simulation, we have not been able to resolve steps at conductances larger than $G=3$. 

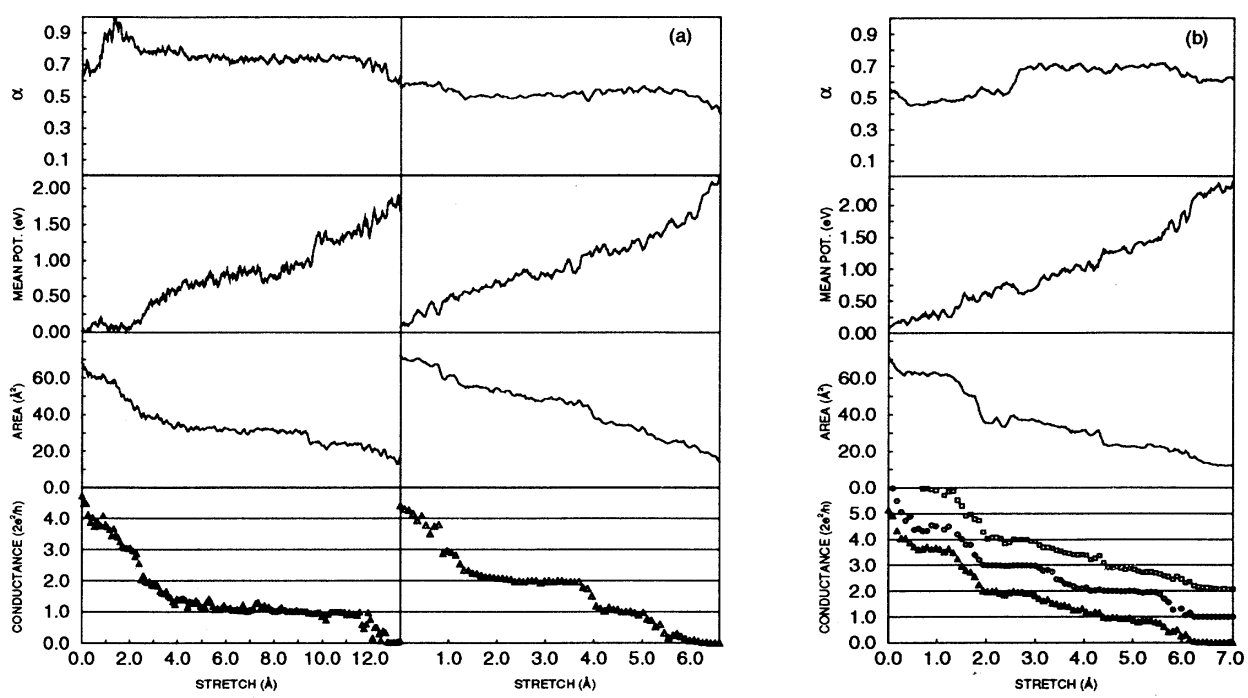

FIG. 13. (a) Two "indentation curves" from the simulation series. The evolution of shape, change in mean potential (due to the increasing surface part of the potential with stretch) and the minimum area at the constriction are shown along with the conductivity. It is seen how changes in these quantities influence the conductivity. (b) An indentation curve obtained in a simulation where scattering dominates the picture $(\triangle)$. The transition from $G=1$ to $G=2$ is almost washed out and a noninteger step appears. We have shown the same simulation scaling $R_{\text {eff }}$ by $5(\bigcirc)$ and by $1 / 5(\square)$. These curves have for clarity been moved up $G+1$ and $G+2$, respectively.

Part of the reason for the "washing out" of steps can be understood from the scaling due to scattering described in Eq. (23), where we see that for a constant radius of curvature of the neck, the widths of the steps increase with the minimum cross-sectional area of the neck as $A^{0.75}$. This scaling is illustrated in the lower histogram in Fig. 14(b), obtained from the expression in Eq. (21), where we have taken a simple model with a linear dependence of minimum cross-sectional area with stretch and an effective curvature of the constriction $R_{\text {eff }}=8 \AA$. We see that we can "fit" the $G=1, G=2$, and $G=3$ peaks in the experimental histogram quite well to the simple model by using a constant mean eccentricity, $\langle\alpha\rangle=0.75$ (also including $\alpha \pm 0.05, \alpha \pm 0.1$ in the statistics as in the simulation). The "fit" is very sensitive to the choice of $\alpha$, as can be seen from the dashed histogram in Fig. 14(b), where we have used $\langle\alpha\rangle=0.8$.

In Fig. 15, we show the area below each of the peaks $G=1,2$, and 3 (scaled to equal the experimental one at $G=1$ ) and the scaling of the widths for the simple model with $\langle\alpha\rangle=0.75$ compared to experiment. We note that for the simple model, we cannot resolve the $G=4$ peak due to the chosen eccentricity, but $G=5$ and even higher can be resolved. This is in contrast to the experimental findings. In the simple model, we have neglected all sorts of mechanical details influencing the evolution of the structure of the neck and resulting, in our simple picture, in a changing $R_{\text {eff }}$ and $\alpha$ during the stretch. We can then conclude that in order to understand the "washing out," these features must be included, as we have done in the simulation.

The histogram corresponding to the simulations is shown in Fig. 14(a). The conductance has been sampled at equidistant times along the stretching process, and we use the same bin width to number of counts ratio as in the experimental histogram, so comparison is possible. We have compared the area and the width of the peaks with the experimental results in Fig. 15. We see that the scaling in the area is in reasonable agreement with the experiment. The number of data points in the peaks in the simulation is less than seen in the experiment. The widths of the two first peaks found in the simulation are a little less compared to the ones found in the experiment, whereas the width of the last $G=3$ is somewhat larger, but still of the right order of magnitude. The difference in width for the first two peaks could be explained by the other scattering sources present in the experiment such as interchannel scattering, scattering at the entrance and exit of the structure and inelastic scattering, which are not included in our model. The reason for larger absolute difference in width for the last $G=3$ peak could be of statistical nature in the sense that we sample the behavior of the constriction by the relatively small number of wires used in the simulation compared to the number of indentations performed in the experiment. This could also be the reason for the smaller number of counts in the peaks found in the simulation compared to the experiment.

\section{CONCLUSION}

We have presented new experimental results with a detailed statistical analysis, which clearly show that for $\mathrm{Au}$ nanowires, the conductance is quantized in quanta of $2 e^{2} / h$. The statistical analysis shows that there is some scatter around the integer values, and this scatter increases with the quantum number, so that only quanta up to three can be clearly identified. The main questions for the subsequent theoretical discussion have, therefore, been why the conductance is quantized, why the quanti- 

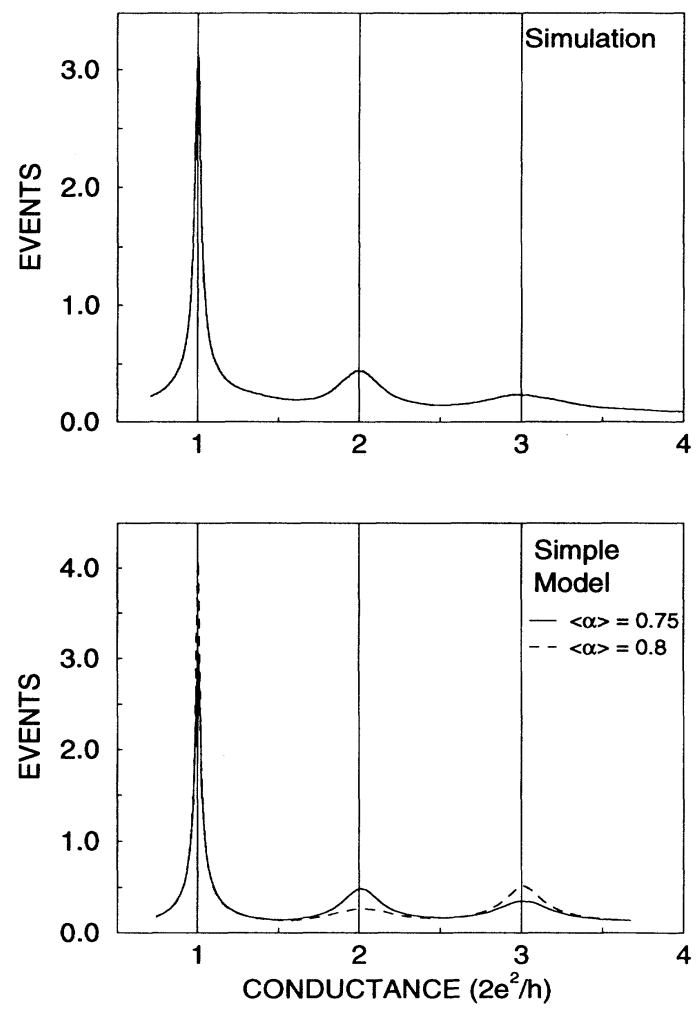

FIG. 14. Histogram from the simulations is shown in (a). For each simulation, the conductance curves with $\alpha, \alpha \pm 0.05$, and $\alpha \pm 0.1$ are included in the statistics. The bin width to number of points sampled is chosen to be the same as in the experimental histogram. In (b) are histograms from a simple model shown. They are obtained from expression (21) assuming equidistant sampling with area, i.e., an area linearly decreasing with the stretch. We have used a constant effective curvature of the constriction of $R_{\text {eff }}=8 \AA$. The full curve is a histogram where we have taken $\alpha=0.75$ and included $\alpha \pm 0.05$ and $\alpha \pm 0.1$ in the statistics $(\langle\alpha\rangle=0.75)$. In the dashed histogram, we have used $\alpha=0.8$. Thus, all mechanical features are neglected in the simple model.

zation is not destroyed by scattering from the boundary of the constriction, and why scattering increases with the number of quanta.

In an adiabatic model, where the cross section of the nanowire varies slowly along the wire, the quantization of the electron motion perpendicular to the wire naturally leads to a quantization of the conductance in units of $2 e^{2} / h$. We have extended this picture by including the elastic scattering from the boundary of the constriction in a free-electron model. In this way, we can account for the experimentally observed conductance histogram both with respect to the relative intensities of the observed peaks and to the increase of the widths for higher numbers of conductance quanta. We argue that the increase of the widths is determined by two factors: The crosssectional area and the effective radius of curvature at the constriction. It has been shown that a simple model, where the effective radius of curvature is kept constant, can be "fitted" to the experiment by choosing a mean eccentricity $(\langle\alpha\rangle=0.75)$ close to the typical value seen

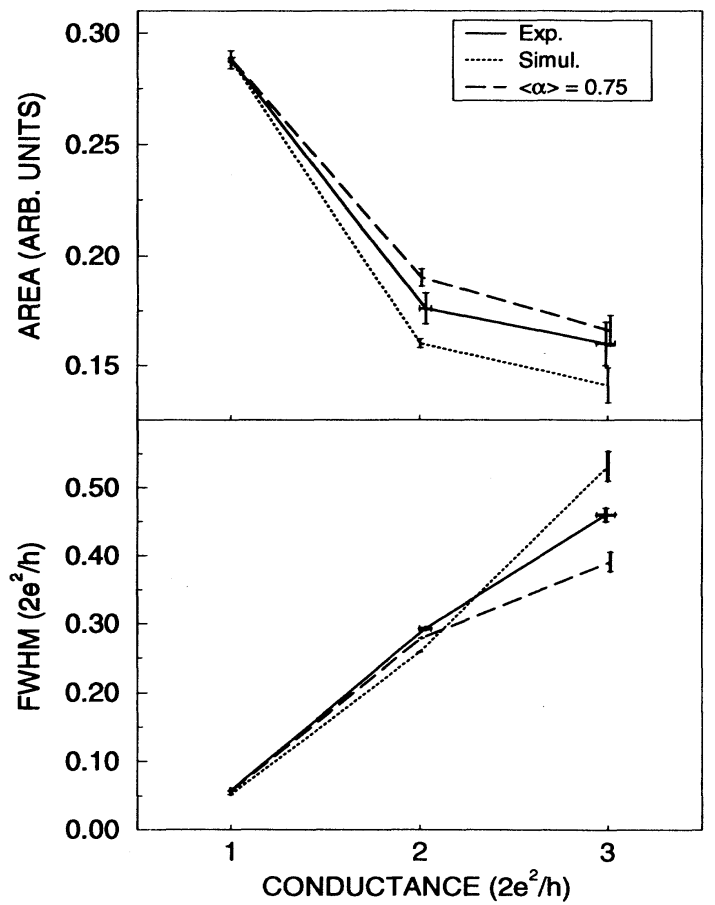

FIG. 15. The area (a) and full width at half maximum (b) in each of the peaks in the histograms of the experiment, simulation, and the simple model with $\langle\alpha\rangle=0.75$. (The theoretical histograms have been scaled so that the area of the peak at one conductance unit is the same as in the experimental histogram.)

in the simulation. However, the simple model fails in two respects: The smearing of the quantum steps bigger than 4 is not described (steps at $G=5$ and higher can be observed in the simple model). It is very sensitive to changes in the choice of mean eccentricity, as we have seen in Fig. 14(b).

This shows that the evolution of the structure close to the constriction during stretch is important and hence that the interplay between modeling of the electronscattering process and atomistic simulations is crucial. Including the evolution of the detailed atomic structures in the conductance model leads to a much improved comparison to the experiments.

We have seen that the reason that scattering from the boundary is not strong enough to completely destroy the quantization of the conductance is that the boundary is quite smooth; the typical effective radius of curvature is about five times the atomic radius. However, it is also clear from the modeling that only a small increase in the scattering will cause the integer steps in the conductance curves to disappear. If, for instance, the system has scattering centers around the constriction with a typical length scale, which is more like an atomic radius, then tunneling and reflection will be so dominating that the quantized conductance will disappear [cf. Fig. 13(b)]. In that case, the conductance is found always to reflect the variations in the cross-sectional area of the constriction, and the jumps in conductance are closely related to the mechanical instabilities that we observe in our sim- 
ulations [cf. Fig. 13(b)]. This may still lead to conductance plateaus, but now there is no reason why they are at integer values of $2 e^{2} / h$. This is the behavior that we expect for large conductances, where the scattering becomes large even with the effective radii of curvature found for the Au constrictions.

The close relation between conductance jumps and mechanical instabilities exists to a certain extent also in the quantized regime. Here, the conductance is still related to the cross-sectional area even though not in a smooth monotonic way. Large changes in the area are bound to give changes in the conductance as observed in Figs. 8, 13(a), and (b). The main difference between the scattering-dominated and the quantized regimes is that in the latter case, the quanta are fixed at integer values of the quantum conductance unit, whereas there is no reason why that should be the case in the other regime. It is, therefore, not surprising that both calculations and experiments show a correlation between mechanical instabilities and the conductance.

There are still many questions to be answered before we have a complete understanding of the electrical and mechanical properties of point contacts between two metals. Let us mention just a few. It is not exactly clear how impurities present in the nanowire and phonons af- fect the conductance quantization. Experiments where controlled amounts of adsorbates are added to the system may be important here. Along these lines, it is also of importance to examine the differences in the conduction quantization between different metals. Regarding the theoretical modeling, we need to improve the theoretical description of the conductance. Hereto only quite approximate methods based on the free-electron model or tight binding model have been used. Among the mechanical properties, we still do not have a complete understanding of the atomic relaxations involved when the contact is formed, i.e., the "jump to contact," and during the stretch. In the latter case, there are currently contradictions between the experiment by Stalder and Dürig $^{19}$ and the molecular dynamics simulations in this paper and in Ref. 12, as mentioned in Sec. III. Finally, in the experiments by Krans et al. ${ }^{21}$ there are signs of hysteresis that also need further understanding.

\section{ACKNOWLEDGMENTS}

The Center for Atomic-scale Materials Physics is sponsored by the Danish National Research Foundation. Further funding is obtained from the Danish Research Councils through the Center for Nano-Tribology.
${ }^{1}$ Y. V. Sharvin, Zh. Eksp. Teor. Fiz. 48, 984 (1965) [Sov. Phys. JETP 21, 655 (1965)].

${ }^{2}$ A. G. M. Jansen, A. P. van Gelder, and P. Wyder, J. Phys. C 13, 6073 (1980).

${ }^{3}$ D. A. Wharam, T. J. Thornton, R. Newbury, M. Pepper, H. Ahmed, J. E. F. Frost, D. G. Hasko, D. C. Peacock, D. A. Ritchie, and G. A. C. Jones, J. Phys. C 21, L209 (1988).

${ }^{4}$ B. J. van Wees, H. van Houten, C. W. J. Beenakker, J. G. Williamson, L. P. Kouwenhoven, D. van der Marel, and C. T. Foxon, Phys. Rev. Lett. 60, 848 (1988).

${ }^{5}$ For a recent review, see C. W. J. Beenakker and H. van Houten, in Solid State Physics: Advances in Research and Applications, edited by H. Ehrenreich and D. Turnbull (Academic, New York, 1991), Vol. 44.

${ }^{6}$ G. Binnig and H. Rohrer, Rev. Mod. Phys. 59, 615 (1987).

${ }^{7}$ See, e.g., Atomic and Nanometer-Scale Modifications of Materials: Fundamentals and Applications, Vol. 239 of NATO Advanced Study Institute Series E: Applied Sciences, edited by P. Avouris (Kluwer, Dordrecht, 1992).

${ }^{8}$ U. Dürig, O. Züger, and D. W. Pohl, Phys. Rev. Lett. 65, 349 (1990).

9 J. B. Pethica and A. P. Sutton, J. Vac. Sci. Technol A 6, 2490 (1988).

${ }^{10}$ J. K. Gimzewski and R. Möller, Phys. Rev. B 36, 1284 (1987).

11 N. D. Lang, Phys. Rev. B 36, 8173 (1987).

${ }^{12}$ U. Landman, W. D. Luedtke, N. A. Burnham, and R. J. Colton, Science 248, 454 (1990).

${ }^{13}$ N. Agraït, J. G. Rodrigo, and S. Vieira, Phys. Rev. B 47, 12345 (1993).

${ }^{14}$ N. Agraït, J. G. Rodrigo, C. Sirvent, and S. Vieira, Phys. Rev. B 48, 8499 (1993).

15 J. I. Pascual, J. Méndez, J. Gómez-Herrero, A. M. Baró, N. García, and Vu Thien Binh, Phys. Rev. Lett. 71, 1852
(1993).

16 J. I. Pascual, J. M. Méndez, J. Gómez-Herrero, A. M. Baró, N. Garcia, U. Landman, W. D. Luedtke, E. N. Bogachek, and H. P. Cheng, Science, 267, 24 (1995).

${ }^{17}$ V. V. Dremov, S. Yu. Shapoval, and E. V. Sukhorukov, Phys. Low-Dim. Struct. 11/12, 29 (1994).

${ }^{18}$ L. Olesen, E. Lægsgaard, I. Stensgaard, F. Besenbacher, J. Schiøtz, P. Stoltze, K. W. Jacobsen, and J. K. Nørskov, Phys. Rev. Lett 72, 2251 (1994).

19 A. Stalder and U. Dürig (unpublished).

${ }^{20}$ C. J. Muller, J. M. van Ruitenbeek, and L. J. de Jongh, Phys. Rev. Lett. 69, 140 (1992).

${ }^{21}$ J. M. Krans, C. J. Muller, I. K. Yanson, Th. C. M. Govaert, R. Hesper, and J. M. van Ruitenbeek, Phys. Rev. B 48, 14721 (1993).

${ }^{22}$ T. N. Todorov and A. P. Sutton, Phys. Rev. Lett. 70, 2138 (1993).

23 J. A. Torres, J. I. Pascual, and J. J. Sáenz, Phys. Rev. B 49, 16581 (1994).

${ }^{24}$ C. Klink, L. Olesen, F. Besenbacher, I. Stensgaard, E. Lægsgaard, and N. D. Lang, Phys. Rev. Lett. 71, 4350 (1993).

${ }^{25}$ F. Besenbacher, E. Lægsgaard, L. Pleth Nielsen, L. Ruan, and I. Stensgaard, J. Vac. Sci. Technol. B 12, 1758 (1994).

${ }^{26}$ L. Eierdal, F. Besenbacher, E. Lægsgaard, and I. Stensgaard, Surf. Sci. 312, 31 (1994).

${ }^{27}$ G. Binnig, N. García, H. Rohrer, J. M. Soler, and F. Flores, Phys. Rev. B 30, 4816 (1984).

${ }^{28}$ N. D. Lang, Phys. Rev. B 37, 10395 (1988).

${ }^{29}$ K. W. Jacobsen, J. K. Nørskov, and M. J. Puska, Phys. Rev. B 35, 7423 (1987); P. Stoltze, J. Chem. Phys. 92, 6306 (1990).

${ }^{30}$ K. W. Jacobsen, Comments Condens. Matter Phys. 14, 129 (1988). 
${ }^{31}$ K. Nikolić and A. MacKinnon, Phys. Rev. B 50, 11008 (1994).

${ }^{32}$ R. Landauer, IBM J. Res. Dev. 1, 223 (1957).

${ }^{33}$ M. Büttiker, Y. Imry, R. Landauer, and S. Pinhas, Phys. Rev. B 31, 6207 (1985).

${ }^{34}$ O. Jepsen, D. Glötzel, and A. R. Mackintosh, Phys. Rev. 23, 2684 (1981).

${ }^{35}$ Bjørk Hammer (private communication).

${ }^{36}$ A. O. E. Animalu, Phys. Rev. B 8, 3542 (1973).

${ }^{37}$ E. C. Kemble, The Fundamental Principles of Quantum Mechanics with Elementary Applications (Dover, New York, 1937); B. S. Jeffreys, in Quantum Theory, edited by D. R. Bates (Academic Press, New York, 1961), p. 229.

${ }^{38}$ L. I. Glazman, G. B. Lesovik, D. E. Khmel'nitskii, and R.
I. Shekhter, Pis'ma Zh. Eksp. Teor. Fiz. 48, 218 (1988) [JETP Lett. 48, 238 (1988)].

39 A. Yacoby and Y. Imry, Phys. Rev. B 41, 5341 (1990).

${ }^{40}$ A. Stern, Y. Aharonov, A. Yacoby, and Y. Imry, in Proceedings of the Third International Symposium on Foundations of Quantum Mechanics in the Light of New Technology (Physical Society of Japan, Tokyo, 1990), pp. 201-211.

${ }^{41}$ F. A. Maa $\varnothing$, I. V. Zozulenko, and E. H. Hauge, Phys. Rev. B 50, 17320 (1994).

42 J. C. Maxwell, Treatise on Electricity and Magnetism (Clarendon, Oxford, 1904).

43 G. Wexler, Proc. Phys. Soc. 89, 927 (1966).

${ }^{44}$ See, e.g., I. -W. Lyo and Ph. Avouris, Science 253, 173 (1991). 


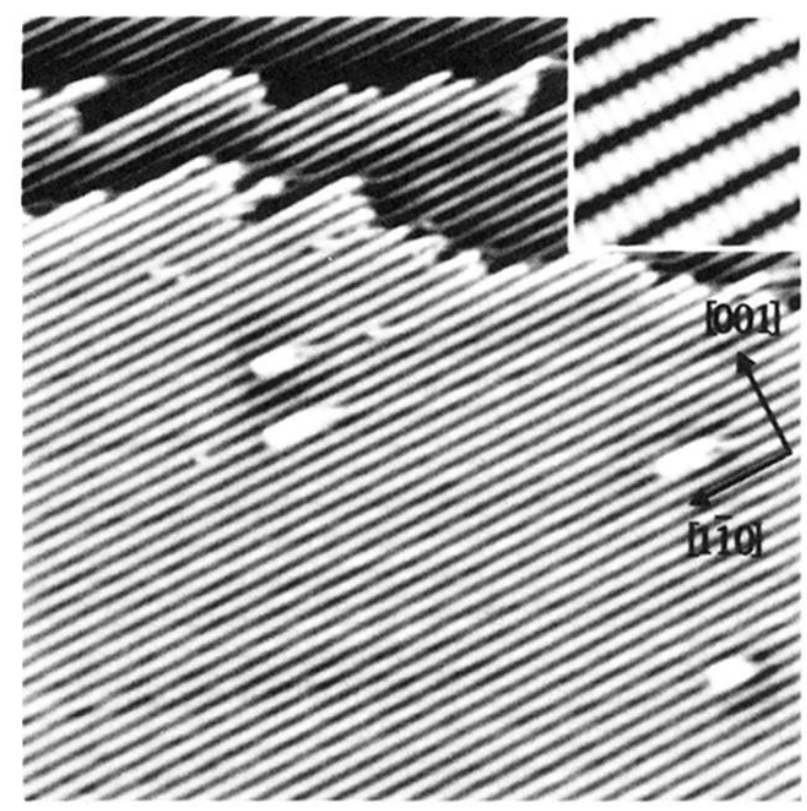

FIG. 1. A $325 \times 335 \AA^{2}$ constant-current image of the clean, $(1 \times 2)$ reconstructed $\mathrm{Au}(110)$ surface recorded prior to an indentation with tunneling parameters $I_{t} \approx 4 \mathrm{nA}$ and $V_{t} \approx 3 \mathrm{mV}$. The inset shows a $40 \times 40 \AA^{2}$ closeup of the rows running along the $[1 \overline{1} 0]$ direction. 